\title{
A rapid and simple method for constructing stable mutants of Acinetobacter baumannii
}

\author{
Jesús Aranda ${ }^{1 \dagger}$, Margarita Poza ${ }^{1 \dagger}$, Belén G Pardo², Soraya Rumbo ${ }^{1}$, Carlos Rumbo ${ }^{1}$, José R Parreira', \\ Patricia Rodríguez-Velo', Germán Bou ${ }^{1 *}$
}

\begin{abstract}
Background: Acinetobacter baumannii is a multidrug-resistant bacterium responsible for nosocomial infections in hospitals worldwide. Study of mutant phenotypes is fundamental for understanding gene function. The methodologies developed to inactivate A. baumannii genes are complicated and time-consuming; sometimes result in unstable mutants, and do not enable construction of double (or more) gene knockout mutant strains of A. baumannii.
\end{abstract}

Results: We describe here a rapid and simple method of obtaining A. baumannii mutants by gene replacement via double crossover recombination, by use of a PCR product that carries an antibiotic resistance cassette flanked by regions homologous to the target locus. To demonstrate the reproducibility of the approach, we produced mutants of three different chromosomal genes (omp33, oxyR, and soxR) by this method. In addition, we disrupted one of these genes (omp33) by integration of a plasmid into the chromosome by single crossover recombination, the most widely used method of obtaining A. baumannii mutants. Comparison of the different techniques revealed absolute stability when the gene was replaced by a double recombination event, whereas up to $40 \%$ of the population reverted to wild-type when the plasmid was disrupting the target gene after 10 passages in broth without selective pressure. Moreover, we demonstrate that the combination of both gene disruption and gene replacement techniques is an easy and useful procedure for obtaining double gene knockout mutants in A. baumannii.

Conclusions: This study provides a rapid and simple method of obtaining stable mutants of $A$. baumannii free of foreign plasmidic DNA, which does not require cloning steps, and enables construction of multiple gene knockout mutants.

\section{Background}

Acinetobacter baumannii is a Gram-negative coccobacillus that is increasingly recognized as a major pathogen causing nosocomial infections worldwide, particularly in patients admitted to intensive care units [1,2]. A. baumannii can cause pneumonia, wound infections, urinary tract infections, bacteremia and meningitis [3,4]. Its clinical significance, especially in recent years, has increased because of the ability of the bacterium to acquire resistance determinants, making it one of the microorganisms threatening the current antibiotic era [5].

\footnotetext{
* Correspondence: german.bou.arevalo@sergas.es

+ Contributed equally

'Servizo de Microbioloxía-INIBIC, Complexo Hospitalario Universitario A

Coruña, As Xubias s/n, 15006. A Coruña, Spain Full list of author information is available at the end of the article
}

The availability of the genome sequences of several strains of $A$. baumannii opens up new perspectives in the study of this bacterial species [6-9]. The artificial introduction of mutations, by molecular techniques, is a useful way of advancing our understanding of the genetics of A. baumannii. The method most commonly used to generate $A$. baumannii mutants involves integration of a plasmid into the chromosome by single crossover recombination. This method requires an internal fragment homologous to the target gene cloned into a suicide vector carrying resistance cassettes [10], which is a major limitation for systematic construction of mutants in post-genomic studies of $A$. baumannii. The possibility that a second crossover event will return the mutant to a wild-type phenotype is another important inconvenience. The gene replacement method is a useful way of overcoming these limitations.
C Biomed Central 
Gene replacement typically involves transformation of a non-replicating plasmid containing a deleted or modified gene, followed by low-frequency integration of a plasmid into the chromosome and selection for resolution events to identify gene replacement candidates. In fact, in A. baumannii, the plasmids pSSK10, pEX100T, and pJQ200 are valuable tools for constructing mutants by this methodology [11-13]. However, these gene replacement methodologies require several subcloning steps and phenotypic screenings. As a means of circumventing these complicated approaches, we have developed a rapid and simple method of inactivating of chromosomal genes that does not require cloning steps. Moreover, the mutants grow directly on agar plates containing appropriate antibiotics and are confirmed by a simple PCR assay.

Integration of a linear piece of foreign DNA requires two recombination events, whereby the original genetic material is replaced by the recombinant DNA [14]. The methodology used in the present study is based on electroporation of a recipient $A$. baumannii strain with a linear PCR fragment carrying an antibiotic resistance cassette flanked by regions homologous to the target locus. This method was used successfully to inactivate three chromosomal loci in A. baumannii (omp33, oxyR, and $\operatorname{sox} R$ ). Moreover, the stability of the mutant, gene expression, and the efficiency of gene disruption and gene replacement methods were compared. Finally, the combination of both techniques was found to be an easy and useful method of obtaining double knockout mutants of $A$. baumannii.

\section{Results}

Replacement of the A. baumannii omp33 gene

A PCR product containing a kanamycin resistance cassette flanked by $500 \mathrm{bp}$ of the regions surrounding the omp33 gene (Figure 1a, Table 1) was introduced into the A. baumannii ATCC 17978 strain by electroporation. After selection on kanamycin-containing plates, the A. baumannii $\Delta$ omp33::Km mutant was obtained. The frequency of generation of mutants by gene replacement was approximately $10^{-7}$. The PCR tests with locus-specific primers revealed that 2 of 15 clones obtained had replaced the wild-type gene by the kanamycin cassette (Figure 1b). In addition, allelic replacement in mutant clones was further confirmed by sequencing the PCR products obtained (data not shown).

\section{Disruption of the A. baumannii omp33 gene}

The gene disruption method was also used to inactivate the omp33 gene. Gene disruption was carried out by cloning a 387-pb internal fragment of the omp33 gene into the pCR-BluntII-TOPO, to obtain the pTOPO33int plasmid (Figure 2a). After transformation of the recombinant plasmid into the $A$. baumannii ATCC 17978 strain and selection on kanamycin-containing plates, the A. baumannii omp33::TOPO mutant was obtained. The frequency of generation of mutants by gene disruption was approximately $10^{-5}$. PCR tests with locus-specific primers revealed that all the clones analyzed (10 of approximately 100) contained fragments of the expected size (Figure $2 \mathrm{~b}$ ). In addition, gene disruption in mutant clones was further confirmed by sequencing the PCR products obtained (data not shown).

\section{Stable maintenance of plasmid insertion into the chromosome requires drugselection}

Gene knockout stability was tested by culturing both the $\Delta$ omp33::Km and omp33::TOPO A. baumannii mutants under nonselective conditions (in the absence of antibiotics). Cultures of the mutant strains were initially grown in LB and at passages 1, 5, and 10, the cultures were dilution plated to obtain individual colonies, with replicate platings of 100 colonies for each strain on LB and LB supplemented with kanamycin. The frequency of loss of kanamycin resistance in each passage after growth in non-selective conditions was 1\% (first), $9 \%$ (fifth), and 37\% (tenth) for the gene disrupted omp33:: TOPO mutant. By contrast, the gene-replaced $\Delta$ omp33:: $\mathrm{Km}$ mutant was stable since no reversions were detected in any passage. As expected, when the same experiment was carried out in the presence of selective pressure, both mutants remained stable (all colonies analyzed were resistant to kanamycin).

\section{Complementation}

Taking advantage of the fact that the Omp33 protein has been identified in the proteome of $A$. baumannii ATCC 17978 strain by 2-DE and MALDITOF/TOF [15], we observed the absence of the Omp33 protein by 2-DE analysis of the $\Delta$ omp33::Km mutant (Figure 3a). In order to complement the mutant phenotype, we constructed and tested the expression plasmid pET-RA. The wild-type omp33 gene without its promoter region was cloned into this expression plasmid. This construction was then introduced into the $\Delta$ omp33::Km mutant strain by electroporation. The cell surface-associated proteins of the wild-type strain and the $\Delta$ omp $33:: \mathrm{Km}$ mutant strain complemented with the pET-RA-OMP33 plasmid were extracted and analyzed by $2 \mathrm{DE}$. The Omp33 protein was detected in the mutant complemented with the Omp33 ORF under the control of the $\beta$-lactamase CTX-M14 gene promoter of the pET-RA plasmid (Figure 3a).

\section{Omp33 detection}

Western blot analysis was performed for further confirmation of the absence of Omp33 in the A. baumannii 
(a)

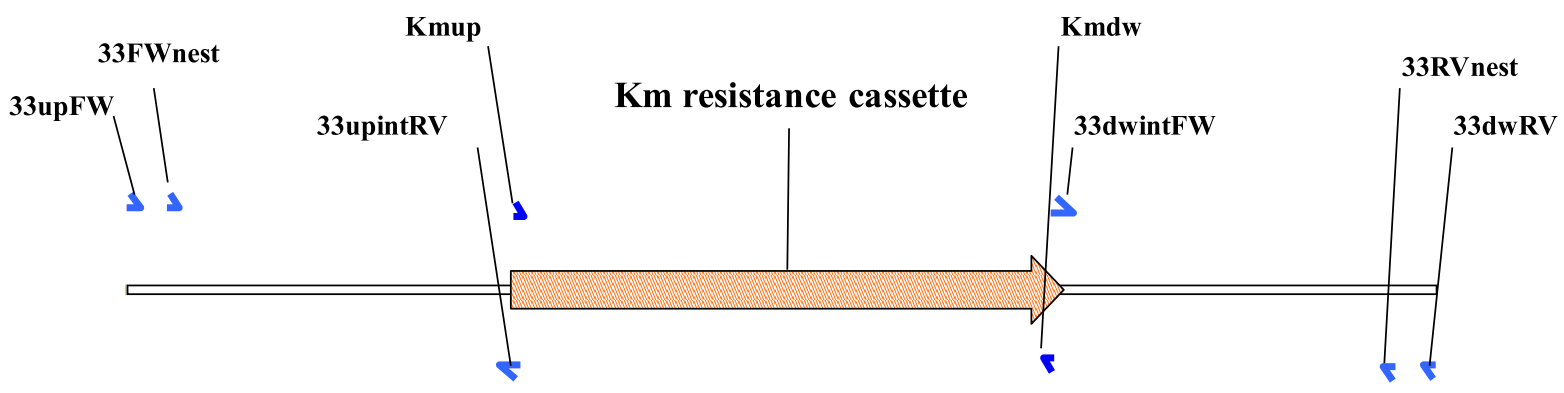

$\Delta$ omp33::Km mutant

(b)

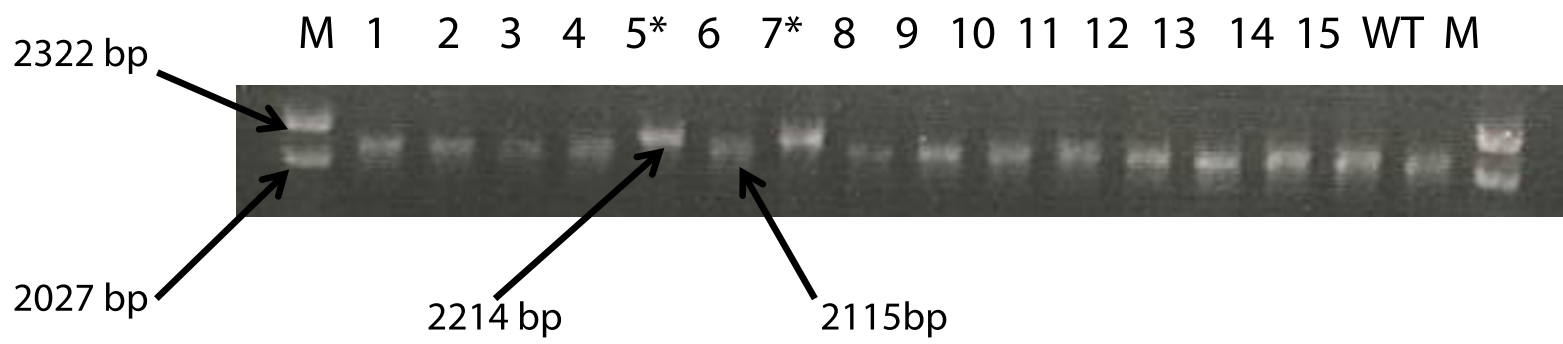

Figure 1 omp33 replacement. (a) Schematic representation of the linear DNA constructed for the omp33 gene replacement, which was completely deleted. The oligonucleotides used (small arrows) are listed in Table 2. (b) Screening of omp33 A. baumannii mutants generated by gene replacement. The numbers at the top are bacterial colony numbers. WT, Wild-type control with $2115 \mathrm{bp}$. Colonies 5 and 7 (lanes $5^{*}$ and $7^{*}$ ) with $2214 \mathrm{bp}$ (2115 bp - $834 \mathrm{bp}$ [from omp33 deletion] + $933 \mathrm{bp}$ [from kanamycin insertion]) were sequenced to confirm gene replacement. Lambda DNA-Hind III and $\varphi$ X174 DNA-Hae III Mix (Finnzymes) was used as a size marker (M). The lengths of PCR products and of some molecular size marker fragments are also indicated.

mutants. For this purpose, cell surface-associated proteins of wild-type strain, omp33 mutants, and pET-RAOMP33-complemented mutants were extracted and subjected to Omp33 Western blot analysis (Figure 3b). The Omp33 protein was not detected in the cell surface-associated proteins of the mutants. As expected, the Omp33 protein was detected in the cell surface- associated proteins of both $\Delta$ omp33::Km and omp33:: TOPO mutants containing the pET-RAOMP33 vector.

\section{Reproducibility of the gene replacement method}

To ensure reproducibility of the gene replacement method, we produced the gene replacements of $o x y R$ and soxR (Table 1). The same gene replacement method

Table 1 Genes of $A$. baumannii strain ATCC 17978 inactivated in the present study

\begin{tabular}{|c|c|c|c|c|}
\hline Product Name & Gene location $^{a}$ & Length $^{\mathbf{b}}$ & Locus tag ${ }^{c}$ & Accession number \\
\hline Outer membrane protein (Omp33) & 3789880 to 3790566 & 228 & A1S_3297 & YP_001086288.1 \\
\hline Transcriptional regulator SoxR & 1547914 to 1548219 & 101 & A1S_1320 & YP_001084350.1 \\
\hline Transcriptional regulator OxyR & 1150365 to 1151153 & 262 & A1S_0992 & YP_001084026.1 \\
\hline
\end{tabular}

\footnotetext{
${ }^{\mathrm{a}}$ A. baumannii ATCC 17978 chromosomal coordinates for each gene.
}

${ }^{\mathrm{b}}$ The length is expressed as number of amino acids.

${ }^{c}$ Based on National Center for Biotechnology Information http://www.ncbi.nlm.nih.gov. 


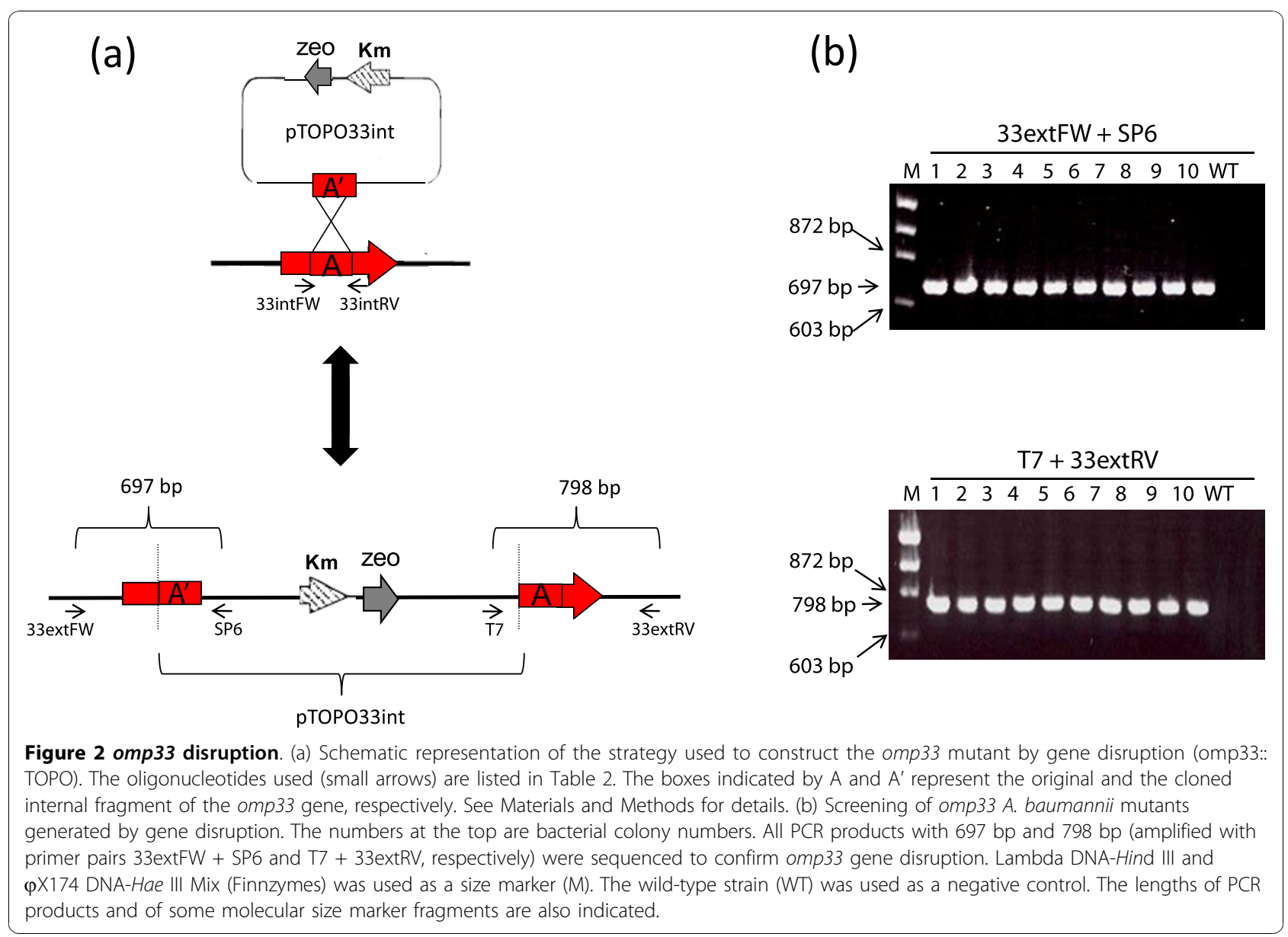

used to produce the $\Delta$ omp33::Km mutant was also used to construct the $\Delta$ oxyR::Km and $\Delta$ soxR::Km mutants (Figure 4), with the primers listed in Table 2. The PCR tests with locus-specific primers revealed that 2 of the 7 clones obtained for the oxyR gene, and all clones (3) obtained for the soxR gene had replaced the wild-type gene with the kanamycin resistance cassette (Figure 4). In addition, allelic replacement in mutant clones was further confirmed by sequencing the PCR products obtained (data not shown). Transcriptional analyses demonstrated the lack of both oxyR and soxR gene expression in the $\Delta$ oxyR:::Km and $\Delta$ soxR::Km mutants, respectively (Figure 5 ).

\section{Construction of double knockout mutants}

With the purpose of generating double knockout mutants, the recombinant plasmid pTOPO33int was transformed into both $\Delta$ oxyR::Km and $\Delta$ soxR::Km mutants. After selection on zeocin- and kanamycin-containing plates, the $\triangle$ oxyR::Km-omp33::TOPO and $\Delta$ soxR::Km-omp33::TOPO A. baumannii double knockout mutants were obtained. PCR tests with locus-specific primers revealed that both mutants had fragments of the expected size (data not shown). In addition, gene disruption in mutant clones was further confirmed by sequencing the PCR products obtained, by transcriptional analyses to detect the oxyR and soxR genes (Figure 5), and by Western blot analyses to detect the omp33 gene (data not shown).

\section{Discussion}

Allelic mutation experiments enable investigation of the functions of many unknown genes identified during the sequencing of entire genomes. A number of methods can be used to inactivate bacterial chromosomal genes. As mentioned above, disruption of the A. baumannii chromosome can be achieved by integration of a plasmid into the chromosome by single crossover recombination [10]. For this purpose, an internal fragment that is homologous to the target gene must be cloned into a non-replicating plasmid carrying at least one antibiotic resistance cassette. However, the stability of this type of mutant must be taken into account, because if the genedisrupted mutant cells are grown in a medium lacking antibiotic pressure, the integrated sequence could be removed, and the disrupted gene could revert to the 


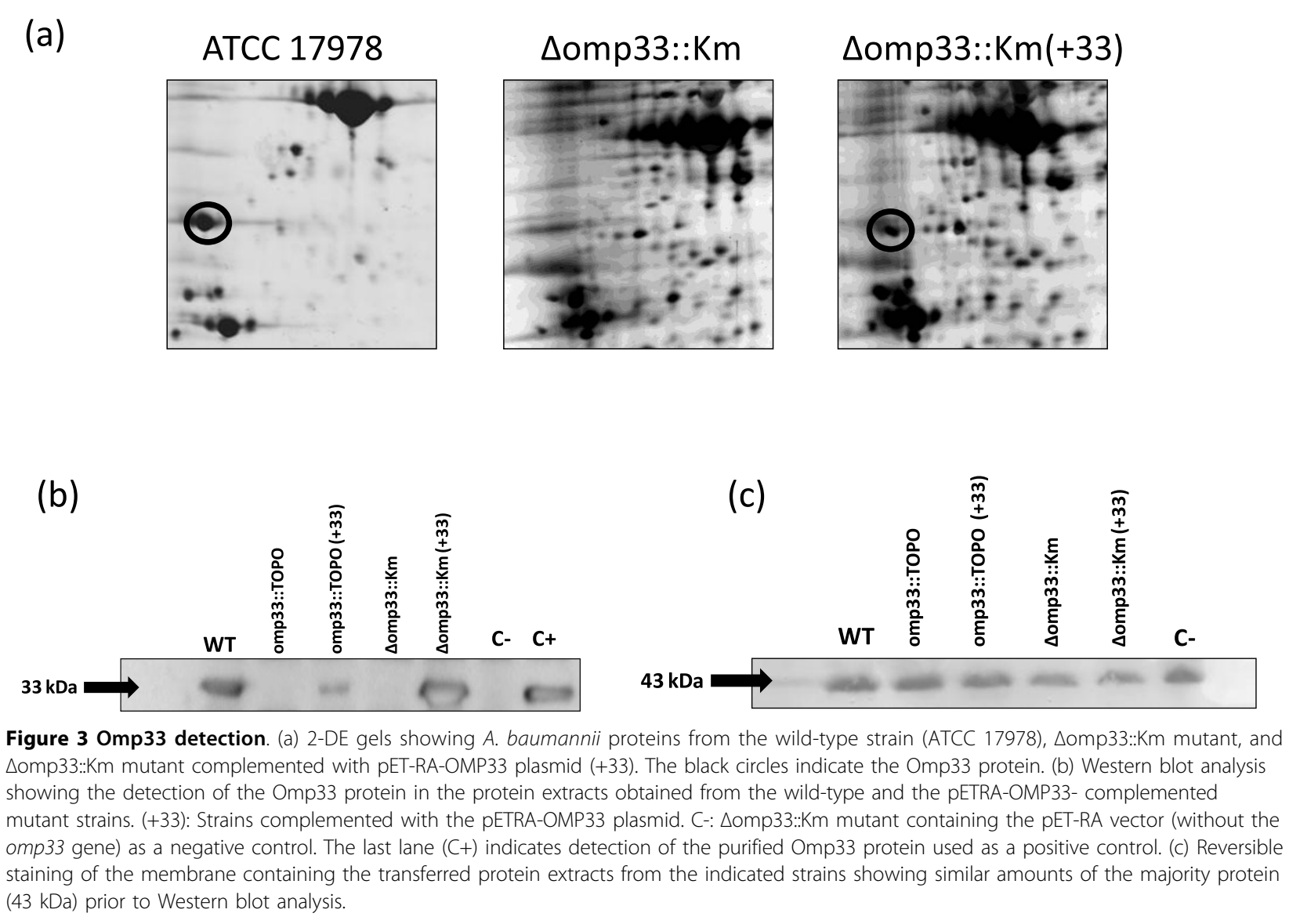

original wild-type [16]. We tested this possibility, and found that this is indeed the case, as also found in similar studies with E. coli [16]. Therefore, one limitation of the method is that the resulting mutants should always be maintained in an appropriate medium containing selective antibiotics. Another disadvantage of the method is that further manipulations of the mutant strain are restricted, because the same vector cannot be used (because undesired recombination events would be highly likely), thus making it impossible to construct multiple gene knockout mutants.

The gene replacement method has recently been used to generate stable A. baumannii mutants [11-13]. This method is based on integration of a plasmid containing the inactivated gene of interest into the bacterial chromosome by single crossover recombination, followed by resolution (or excision) of the integrated DNA by a second recombination event, resulting in replacement of the original wild-type gene by the inactivated gene. The key step in this procedure, in A. baumannii, is counterselection (widely used with Gram-negative bacteria) by the detection of sucrose sensitivity in the presence of the Bacillus subtilis sacB gene coding for levansucrase [17]. This method of counter-selection has been found to be useful for several other environmental bacteria $[11,12,18]$. Plasmids pSSK10, pEX100T, and pJQ200 have been successfully used to obtain $A$. baumannii mutants by this method [11-13]. However, most bacteria subjected to homologous recombination, even under negative selection for the $s a c B$ gene, are wild-type and it is not possible to isolate the desired mutant directly [19-21]. Another disadvantage of this method is that integration of the DNA may not always provide the desired replacement, since foreign DNA with low or no sequence homology would rely on illegitimate recombination events, as previously reported for Acinetobacter and other species $[14,16]$. In addition, all of these gene replacement methodologies are time-consuming, and require several steps involving subcloning into a suicide delivery vector followed by electroporation into $E$. coli and subsequent transfer into A. baumannii by electroporation or conjugation. To avoid such situations, we propose a method based on the electroporation of $A$. baumannii electrocompetent cells with linear DNA, a PCR product including an antibiotic resistance cassette flanked by regions homologous to the target locus. However, as expected, we noted an important disadvantage of the replacement method (which requires two 
(a)

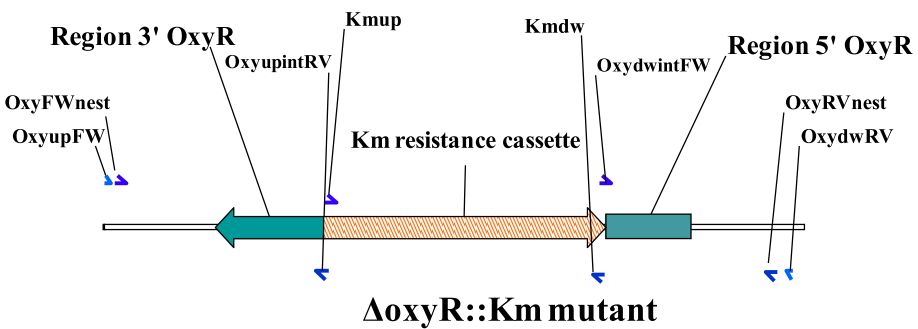

AoxyR::Km mutant (b)

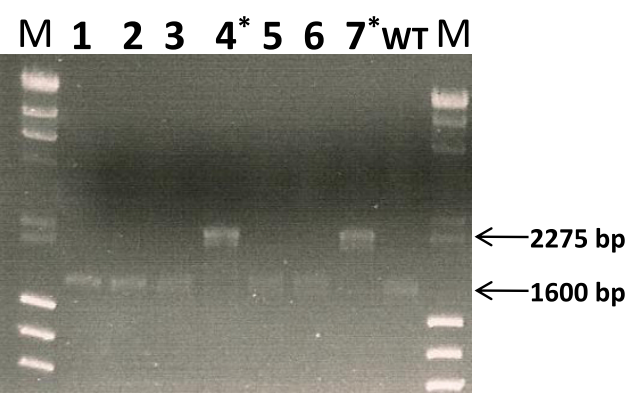

(d)

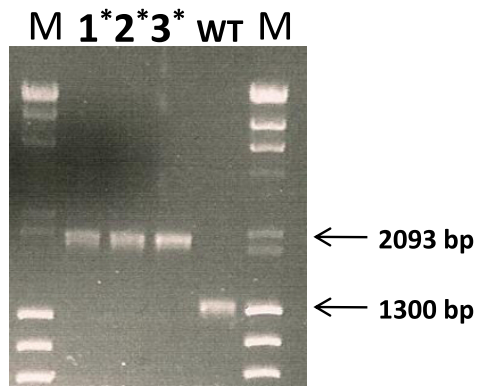

Figure 4 oxyR and soxR replacement. (a) Schematic representation of the linear DNA constructed for the oxyR gene replacement. The oligonucleotides used (small arrows) are listed in Table 2. (b) Screening of oxyR A. baumannii mutants generated by gene replacement. The numbers at the top are bacterial colony numbers. WT; Wild-type control showing 1600 bp. Colonies 4 and 7 (lanes $4^{*}$ and $7^{*}$ ) showing 2275 bp (1600 pb - 258 bp [from oxyR deletion] + 933 bp [from kanamycin insertion]) were sequenced to confirm gene replacement. Lambda DNA-Hind III and $\varphi$ X174 DNA-Hae III Mix (Finnzymes) was used as a size marker (M). (c) Schematic representation of the linear DNA constructed for the soxR gene replacement. The oligonucleotides used (small arrows) are listed in Table 2. (d) Screening of soxR A. baumannii mutants generated by gene replacement. WT: Wild-type control with $1300 \mathrm{bp}$. Colonies 1, 2, and 3 (lanes 1*, 2*, and 3*) with $2093 \mathrm{bp}$ (1300 bp - $140 \mathrm{bp}$ [from soxR deletion] + 933 bp [from kanamycin insertion]) were sequenced to confirm gene replacement. Lambda DNA-Hind III and $\varphi$ X174 DNA-Hae III Mix (Finnzymes) was used as a size marker (M).

recombination events), with respect to the gene disruption method (which only requires one recombination event), i.e. the low efficiency with regard to obtaining mutants $\left(10^{-7}\right.$ vs. $\left.10^{-5}\right)$. In addition, we observed more illegitimate recombination events with the new method than with the gene disruption technique, since several colonies acquired the resistance antibiotic cassette (confirmed by PCR), although the wild-type target gene was not replaced (Figures 1, 2, and 4). Nevertheless, the new method is a useful genetic tool for systematic generation of knockouts. Moreover, to our knowledge, there are no previous reports of double knockout mutant strains of A. baumannii. However, we demonstrate that the combination of both gene disruption and gene replacement techniques is an easy and useful procedure for obtaining double gene-knockout mutants in A. baumannii.

Taking into account the results presented here, it intuitively appears that the gene replacement method would be successful with any strain of A. baumannii, including clinical strains, with the only limitation being the use of an appropriate antibiotic resistance marker. Although the kanamycin resistance cassette cannot be used in clinical strains (all the clinical strains of $A$. baumannii taken from our collection were kanamycin resistant: data not shown), use of another antibiotic resistance marker such as rifampicin (for which a low level of resistance has been demonstrated in approximately $50 \%$ of multidrug-resistant $A$. baumannii clinical isolates in Spain $[22,23])$, would be appropriate for generating mutants by gene replacement in these problematic strains.

\section{Conclusions}

We report mutagenesis of three A. baumannii genes by use of a simple and rapid method. The method offers advantages such as no cloning steps, stability even in the absence of selective pressure, and the possibility of constructing multiple gene knockout mutants. 
Table 2 Oligonucleotides used in the present study

\begin{tabular}{|c|c|c|}
\hline Oligonucleotide name & Sequence $\left(5^{\prime}\right.$ to $\left.3^{\prime}\right)$ & Application \\
\hline 33intUP & CTGGTGACGTTGCTGGTACA & Construction of the omp33::TOPO mutant \\
\hline 33intDW & CGTTACCGATGATACCGAAG & Construction of the omp33::TOPO mutant \\
\hline 33extUP & CCTTAACATTACGTTTCATC & Confirmation of the omp33::TOPO mutant \\
\hline 33extDW & CATGTAAGATGCACCAACTGC & Confirmation of the omp33::TOPO mutant \\
\hline Kmup & CCGGAATTGCCAGCTGGG & Kanamycin amplification \\
\hline Kmdw & TTCAGAAGAACTCGTCAAG & Kanamycin amplification \\
\hline 33upFW & GCTGAGCTCGTAAAGTCTGATG & Construction and confirmation of the $\Delta \mathrm{omp} 33:: \mathrm{Km}$ mutant \\
\hline 33upintRV & CCCAGCTGGCAATTCCGGGGCTAATAATACAGCAGTGG & Construction of the $\triangle$ omp33::Km mutant \\
\hline 33dwintFW & CTTGACGAGTTCTTCTGAAGGCTTAAATGCTAAATTCCG & Construction of the $\Delta$ omp33::Km mutant \\
\hline 33dwRV & CGTTGCCTITTACCGTAGTC & Construction and confirmation of the $\Delta$ omp33:: Km mutant \\
\hline 33FWnest & GCAATTGAATTGTGTGAC & Construction of the $\Delta$ omp33::Km mutant \\
\hline 33RVnest & TGATAGCAATTCAAGAGG & Construction of the $\Delta$ omp33::Km mutant \\
\hline OxyupFW & AGTTAAAAAAAATTGAAGAAA & Construction and confirmation of the $\triangle \mathrm{oxyR}: \mathrm{Km}$ mutant \\
\hline OxyupintRV & CCCAGCTGGCAATTCCGGTCACTTGATGATCTCGATTAA & Construction of the $\triangle \mathrm{oxy} R:: \mathrm{Km}$ mutant \\
\hline OxydwintFW & CTTGACGAGTTCTTCTGAATGAAGATCACCAGTTAATGG & Construction of the $\triangle \mathrm{oxyR}:: \mathrm{Km}$ mutant \\
\hline OxydwRV & TATATTAACCATATTGAAGCC & Construction and confirmation of the $\triangle \mathrm{oxyR}: \mathrm{Km}$ mutant \\
\hline OxyFWnest & GCAACTTGATGCAGCGGT & Construction of the $\triangle \mathrm{oxyR}:: \mathrm{Km}$ mutant \\
\hline OxyRVnest & TCAACGTAGCTACTATCC & Construction of the $\triangle \mathrm{oxyR}:: \mathrm{Km}$ mutant \\
\hline SoxupFW & ATGAAAGAAAAAAACTATATA & Construction and confirmation of the $\Delta$ soxS::Km mutant \\
\hline SoxupintRV & CCCAGCTGGCAATTCCGGATATTTACTTAGGGCTTGTT & Construction of the $\Delta$ soxS::Km mutant \\
\hline SoxdwintFW & CTTGACGAGTTCTTCTGAAGAACAATGTCCATT AGAAA & Construction of the $\Delta$ soxS::Km mutant \\
\hline SoxdwRV & TCTGACTTCGTTITITGCTTA & Construction and confirmation of the $\Delta$ soxS::Km mutant \\
\hline SoxFWnest & AATTGCACGTTGCGATAG & Construction of the $\Delta$ soxS::Km mutant \\
\hline SoxRVnest & TAAACCAGATAGCCCAAC & Construction of the $\Delta$ soxS::Km mutant \\
\hline ATG33Xbal* & ATCGTCTAGACCCAGCTTTATCTCTTGTTA & Cloning of the omp33 gene \\
\hline STOP33Ncol* & ATCGCCATGGGGACTGGACTCAGGAAGATTTG & Cloning of the omp33 gene \\
\hline SoxRTup & AGGAACTGTAATTGCACG & RT-PCR for soxR detection \\
\hline SoxRTrv & CCAATCGAGAGATAGCTC & RT-PCR for soxR detection \\
\hline OxyRTup & ATGGCTGCATTACCCTCACT & RT-PCR for oxyR detection \\
\hline OxyRTrv & GCGCTCTACAATCTTCTCAC & RT-PCR for oxyR detection \\
\hline GyrBup & ATGAGTTCAGAGTCTCAATCA & RT-PCR for gyrB detection \\
\hline GyrBrv & CTGTTAAACCTTCACGCGCAT & RT-PCR for gyrB detection \\
\hline T7 & AATACGACTCACTATAGGG & Universal primer of the pCR-Bluntll-TOPO plasmid \\
\hline SP6 & ATTAGGTGACACTATAG & Universal primer of the pCR-Bluntll-TOPO plasmid \\
\hline pETRAFW & TTCTTCGTGAAATAGTGATGATTIT & Primer of the pET-RA plasmid \\
\hline pETRARV & CTGTTTCATATGATCTGGGTATC & Primer of the pET-RA plasmid \\
\hline
\end{tabular}

*Oligonucleotides including the indicated restriction site (underlined).

The method may therefore facilitate the understanding of the genetics of A. baumannii. Although not tested, it is also possible that this novel method may also work with other pathogenic bacteria, in which genetic manipulation techniques are generally less well established than for E. coli and other bacterial species. Finally, the gene disruption method is recommended when only one $A$. baumannii gene must be inactivated, and when it is possible to maintain selective pressure, since it is the fastest and most efficient method of producing A. baumannii mutants described so far.

\section{Methods}

\section{Bacterial strains, plasmids, and growth conditions}

Bacterial strains and plasmids used in this study are listed in Table 3. The E. coli and A. baumannii strains were grown in Luria Bertani (LB) medium [24]. When necessary, kanamycin $(50 \mu \mathrm{g} / \mathrm{ml})$, rifampicin $(50 \mu \mathrm{g} / \mathrm{ml})$, and zeocin $(20 \mu \mathrm{g} / \mathrm{ml}$ for E. coli and $200 \mu \mathrm{g} / \mathrm{ml}$ for $A$. baumannii) were added to the growth media. All cultures were incubated at $37^{\circ} \mathrm{C}, 180 \mathrm{rpm}$. The frequency of generation of mutants was calculated as the number of mutants obtained, divided by the total CFU. 


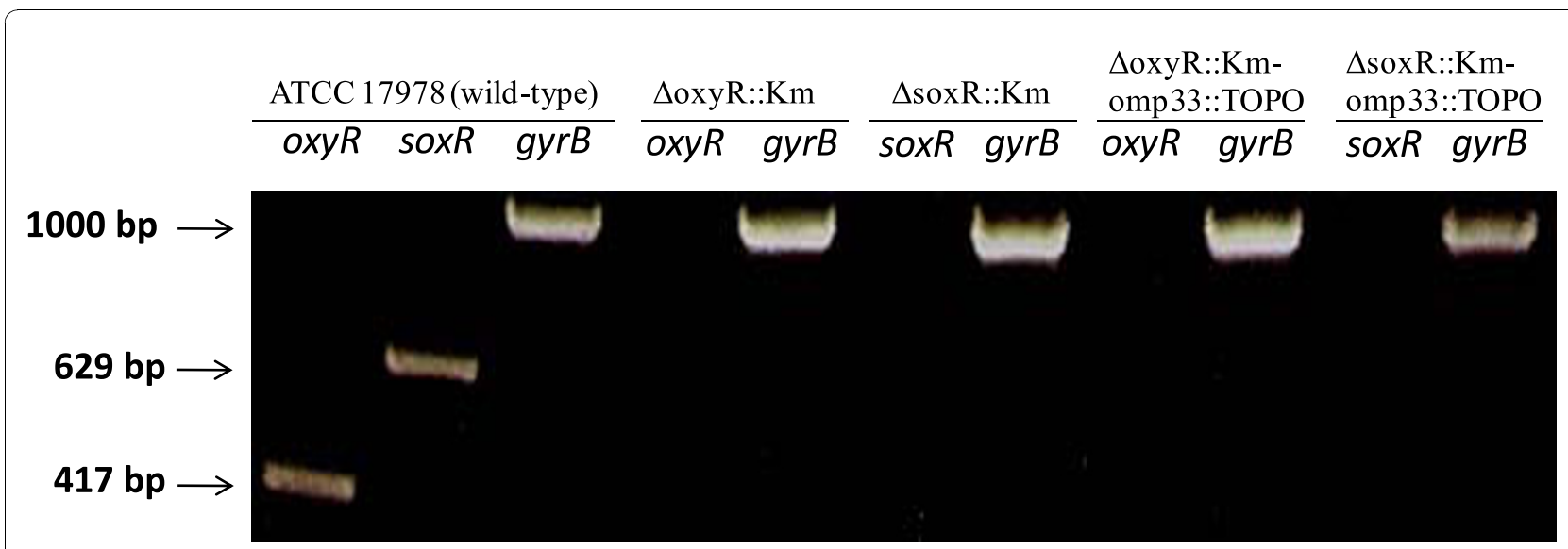

Figure 5 Transcriptional analysis. RT-PCR analysis of RNA extracted from the wild-type, $\triangle$ oxyR::Km, $\triangle$ soxR:::Km, $\triangle$ oxyR:::Km-omp33:::TOPO, and $\triangle$ soxR::Km-omp33::TOPO strains showing the lack of oxyR and soxR transcription in the corresponding mutants. The gyrB gene was used as a housekeeping gene. The lengths of cDNAs obtained are indicated.

\section{DNA manipulations}

Genomic and plasmid isolation were performed with a Wizard Genomic DNA Purification Kit (Promega) and Wizard Plus SV Minipreps DNA Purification System (Promega), respectively. Restriction enzymes and DNAmodifying enzymes were purchased from Promega and used according to the manufacturer's recommendations. Standard PCR amplifications were performed with BioTaq DNA polymerase (Bioline). When necessary, high fidelity and blunt-ended PCR products were amplified with Expand High Fidelity (Roche) and Accuzyme (Bioline) DNA polymerases, respectively. All oligonucleotides (Sigma) used in the study are listed in Table 2. PCR products were purified with the High Pure PCR Product Purification Kit (Roche). When high concentrations of purified PCR products were required, a MinElute PCR Purification Kit (Qiagen) was used. All the recombinant plasmids obtained in the study, and the PCR products indicated, were sequenced by the Macrogen sequencing service (Seoul, Korea).

\section{Electroporation}

All strains were made electrocompetent as follows. Bacterial overnight cultures were grown in LB broth and subcultured at a dilution of 1:20 in $100 \mathrm{ml}$ of fresh LB medium. Cultures were grown at an $\mathrm{OD}_{600}$ of 0.8 and then incubated on ice for $10 \mathrm{~min}$. Cells were pelleted by centrifugation and then washed 3 times with $10 \%(\mathrm{v} / \mathrm{v})$ glycerol and finally resuspended in $500 \mu \mathrm{l}$ of $10 \%(\mathrm{v} / \mathrm{v})$ glycerol. An aliquot of $100 \mu \mathrm{l}$ of the cell suspension was

Table 3 Bacterial strains and plasmids used in the present study

\begin{tabular}{|c|c|c|}
\hline Strain or plasmid & Relevant feature(s) & Source or reference \\
\hline \multicolumn{3}{|l|}{ Strains } \\
\hline \multicolumn{3}{|l|}{ Acinetobacter baumannii } \\
\hline ATCC 17978 & Wild-type strain & Laboratory stock \\
\hline omp33::TOPO & Derived from ATCC 17978. omp33 mutant obtained by plasmid insertion. Kan ${ }^{R}$, Zeo ${ }^{R}$ & Present study \\
\hline$\Delta$ omp33::Km & Derived from ATCC 17978. omp33 mutant obtained by gene replacement. Kan $^{R}$ & Present study \\
\hline$\triangle o x y R:: K m$ & Derived from ATCC 17978. oxyR mutant obtained by gene replacement. Kan $^{R}$ & Present study \\
\hline$\Delta s o x R:: K m$ & Derived from ATCC 17978. soxR mutant obtained by gene replacement. Kan ${ }^{R}$ & Present study \\
\hline$\triangle \mathrm{oxyR}:: \mathrm{Km}$-omp33::TOPO & Derived from $\triangle$ oxyR::Km. oxyR omp33 double mutant. $\operatorname{Kan}^{R}$, Zeo ${ }^{R}$ & Present study \\
\hline$\triangle$ soxR::Km-omp33::TOPO & Derived from $\triangle$ soxR::Km. soxR omp33 double mutant. $\operatorname{Kan}^{R}$, Zeo ${ }^{R}$ & Present study \\
\hline \multicolumn{3}{|l|}{ Escherichia coli } \\
\hline TG1 & supE thi-1 $\Delta($ lac-proAB) $\Delta(m c r B-h s d S M) 5(r K-m K-)$ [F' traD36 proAB laclqZ $\triangle M 15]$ & Laboratory stock \\
\hline \multicolumn{3}{|c|}{ 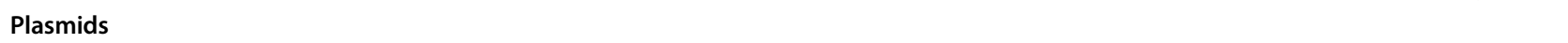 } \\
\hline pCR-BluntII-TOPO & Suicide plasmid for A. baumannii. $\operatorname{Kan}^{R}, Z_{e}{ }^{R}$ & Invitrogen \\
\hline pTOPO33int & pCR-Bluntll-TOPO containing a 387-pb internal fragment of the omp33 gene. $\mathrm{Kan}^{\mathrm{R}}$, Zeo ${ }^{\mathrm{R}}$ & Present study \\
\hline pET-RA & A. baumannii replication origin. CTX-M14 $\beta$-lactamase gene promoter. Rif ${ }^{R}$ & Present study \\
\hline pET-RA-OMP33 & pET-RA containing the omp33 gene without its promoter region. Rif $^{R}$ & Present study \\
\hline
\end{tabular}


mixed with the recombinant DNA (up to $20 \mu \mathrm{l}$ ). The mixture was placed in a pre-chilled sterile electroporation cuvette ( $1 \mathrm{~mm}$ electrode gap, Bio-Rad) and immediately pulsed by use of a Bio-Rad Gene Pulser $(1.8 \mathrm{kV}$, $200 \mathrm{~W}$, and $25 \mu \mathrm{F}$ ). The mixture was incubated at $37^{\circ} \mathrm{C}$ for $1 \mathrm{~h}$ with $1 \mathrm{ml}$ of LB broth. Cells were spread on LB agar containing the appropriate antibiotics and incubated at $37^{\circ} \mathrm{C}$.

\section{Knockout construction by gene replacement}

The upstream and downstream regions (approximately $0.5 \mathrm{kbp}$ each) of the target gene were amplified from genomic DNA of A. baumannii ATCC 17978 strain using primer pairs upFW + upintRV and dwintFW + dwRV (Figure 6), respectively. The kanamycin cassette was amplified using primers Kmup and Kmdw (Table 2) and the pCR-BluntII-TOPO vector (Invitrogen) as a template. The upintRV and dwintFW primers (Figure 6) contained, at their 5' ends, an extension of approximately 20 nucleotides homologous to the Kmup and Kmdw primers, respectively. The three PCR products obtained in the first step were mixed at equimolar concentrations and subjected to a nested overlap-extension PCR with FWnest and RVnest primers (Figure 6) to generate a kanamycin resistance cassette flanked by both the upstream and the downstream gene homologous regions. The nested overlap-extension PCR was carried out with an Expand High Fidelity Taq DNA polymerase (Roche), according to the manufacturer's recommendations; the conditions used were as follows: $94^{\circ} \mathrm{C}$ for $15 \mathrm{~s}, 40^{\circ} \mathrm{C}$ for 1 $\min , 72^{\circ} \mathrm{C}$ for $2 \min \left(10\right.$ cycles); $94^{\circ} \mathrm{C}$ for $15 \mathrm{~s}, 55^{\circ} \mathrm{C}$ for 1 $\min , 72^{\circ} \mathrm{C}$ for $3 \mathrm{~min}$ (20 cycles), and a final extension at $68^{\circ} \mathrm{C}$ for $10 \mathrm{~min}$. Electroporation of the A. baumannii ATCC 17978 strain was performed with approximately 5 $\mu \mathrm{g}$ of the purified PCR product containing the inactivated gene. Cells were then plated on LB agar containing kanamycin for selection of mutants whose wild-type genes were replaced by allelic exchange via double crossover recombination. Gene replacement in candidate clones was verified by PCR with upFW and dwRV primers (Figure 6). Allelic replacement in candidate clones was further confirmed by sequencing the mutant region in the resulting mutants.

\section{Knockout construction by gene disruption}

Plasmid insertion in the omp33 gene (Table 1) was carried out as previously described [10], with slight modifications. Briefly, kanamycin- and zeocin-resistant plasmid pCR-BluntII-TOPO, unable to replicate in A. baumannii, was used as a suicide vector. An internal fragment (387 bp) of the omp33 gene was amplified by PCR with 33intUP and 33intDW primers (Table 2) and genomic DNA from A. baumannii ATCC 17978 as a template. The PCR product was cloned into the pCR-BluntIITOPO vector and electroporated in $E$. coli to yield the pTOPO33int plasmid (Table 3). Recombinant plasmid $(0.1 \mu \mathrm{g})$ was then introduced in the kanamycin- and zeocin-susceptible A. baumannii ATCC 17978 strain by electroporation. Mutants were selected on kanamycincontaining plates. Inactivation of the omp33 gene by insertion of the plasmid via single crossover recombination was confirmed by sequencing the amplified PCR products with the SP6 + 33extUP and T7 + 33extDW primer pairs (Table 2).

\section{Construction of pET-RA plasmid for gene expression in $A$. baumannii}

In order to complement mutant phenotypes, the pETRA plasmid [Genbank: HM219006] was constructed, and carried a rifampicin resistance cassette, a gene

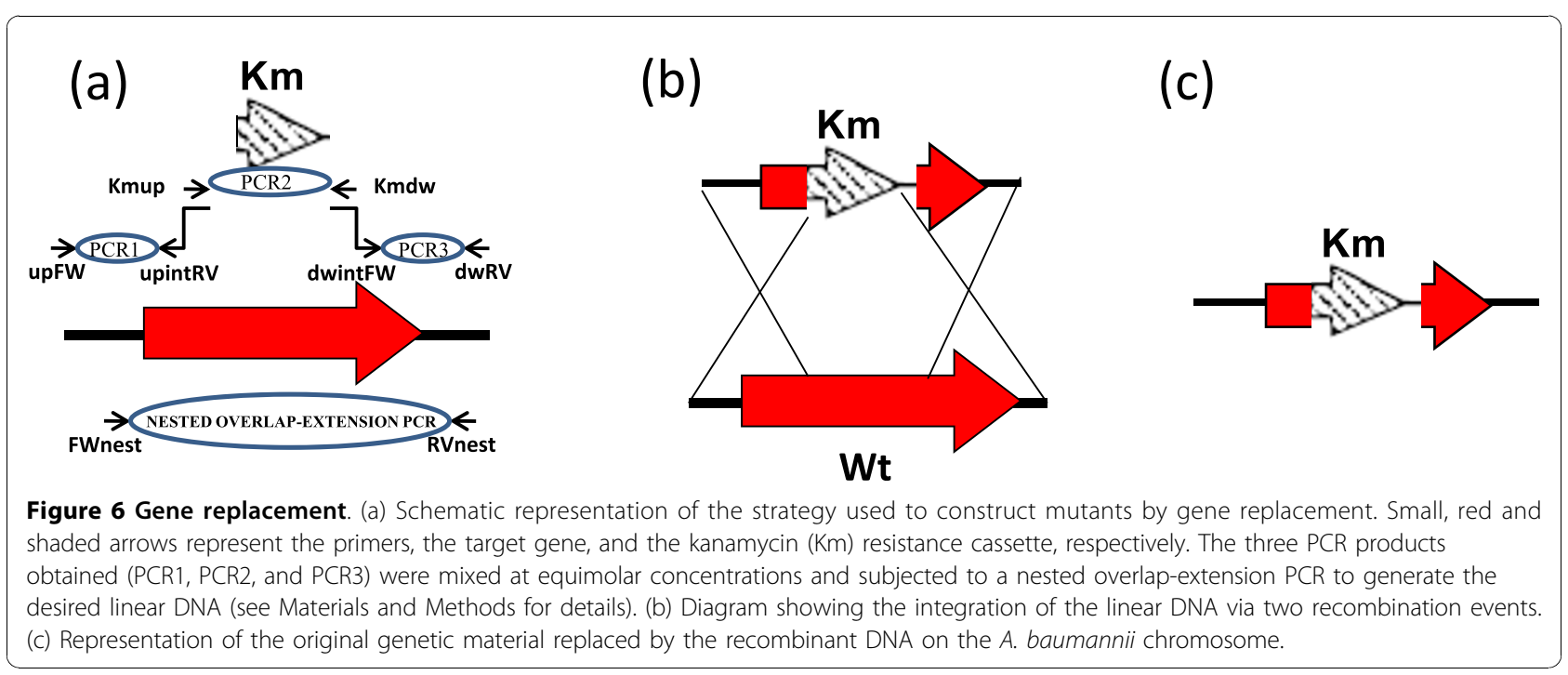


coding for a green fluorescent protein (GFP), and the $A$. baumannii replication origin, which is a plasmid origin of replication (Figure 7). The pET-RA vector was used to express promoterless genes under control of the CTX-M14 $\beta$-lactamase gene promoter, previously cloned upstream of the GFP gene (Figure 7). For pET-RA construction, the pMW82 vector [Genbank: EF363313] was amplified by PCR, excluding the coding region of the ampicillin resistance cassette. The rifampicin resistance cassette was then amplified from the pAT-RA vector [Genbank: HM219005] and introduced into the pMW82 vector. The CTXM-14 promoter was cloned into the pMW82 vector BamHI site. The Acinetobacter replication origin was amplified from the pAT-RA vector and cloned into the HindIII site of the pMW82 vector. As a result, the pET-RA vector containing the CTXM-14 promoter was obtained and used for cloning and expression of genes in the $\mathrm{XbaI}-\mathrm{NcoI}$ restriction sites.

\section{Complementation of omp33 mutants}

In order to complement the A. baumannii omp33 mutants, the omp33 ORF was amplified with the
ATG33XbaI and STOP33NcoI primers (Table 2) from the A. baumannii ATCC 17978 strain genome and cloned into the $X b a \mathrm{I}-\mathrm{NcoI}$ restriction sites of the pET-RA vector under the control of the $\beta$-lactamase CXT-M-14 gene promoter yielding the pET-RA-OMP33 plasmid (Table 3). Acinetobacter baumannii omp33 mutants were transformed with the recombinant pETRA-OMP33 plasmid. Transformants were selected on rifampicin- and kanamycin-containing plates and confirmed by PCR with the PETRAFW and pETRARV primers (Table 2).

\section{Mutant stability assays}

The bacterial cultures were grown in $5 \mathrm{ml}$ of LB broth without kanamycin and incubated at $37^{\circ} \mathrm{C}$. Every day, during 10 consecutive days, $100 \mu \mathrm{l}$ of each culture was diluted in $5 \mathrm{ml}$ of fresh medium and incubated for 24 $h$. The same experiment was also carried out, for each strain, with medium containing kanamycin. On days 1 , 5 , and 10 , all cultures were diluted $10^{6}$-fold and dilutions $(0.1 \mathrm{ml})$ were plated on non-selective plates. From these plates, 100 colonies were each transferred to non-

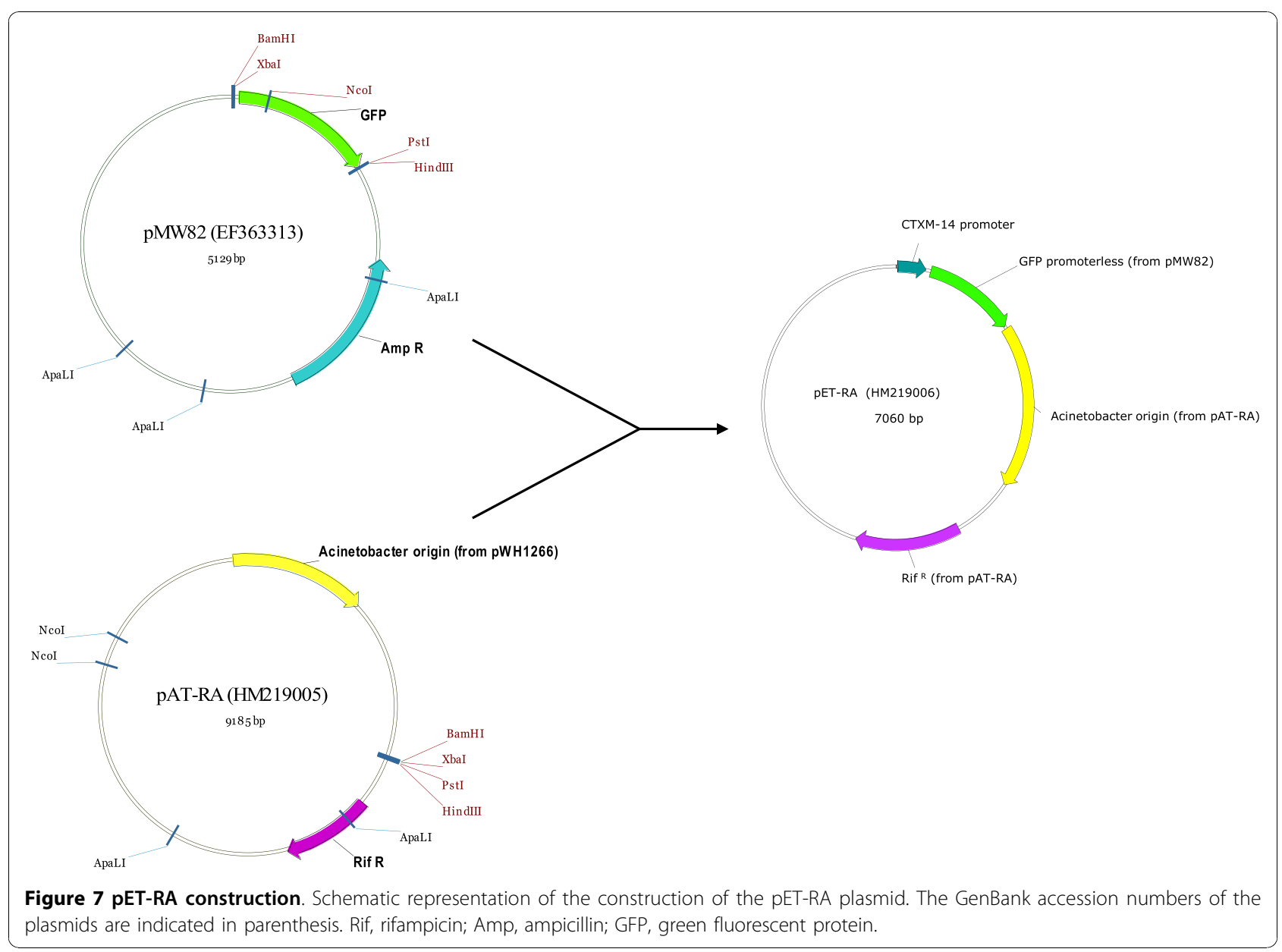


selective and selective plates to determine the frequency of revertants, on the basis of the percentage of kanamycinsusceptible colonies.

\section{RNA methods}

Bacterial cultures were grown overnight in LB broth supplemented with the appropriate antibiotics at $37^{\circ} \mathrm{C}$. RNA isolation was performed with the RNAeasy Plant Mini Kit (Qiagen). The total RNA extraction was subjected to DNaseI (Invitrogen) treatment, following the manufacturer's instructions. In order to evaluate transcription of the genes of interest, an RT-PCR was performed with the First Strand cDNA Transcriptor Synthesis Kit (Roche) and the $\operatorname{gyr} B$ as housekeeping gene. Both the first strand synthesis and the PCR amplification were carried out with the specific primers listed in Table 2. Finally, RT-PCR products were visualized in a $1 \%$ agarose gel.

\section{Protein analysis}

Extraction of A. baumannii cell surface-associated proteins and two-dimensional gel electrophoresis (2-DE) were performed as described elsewhere [15]. Proteins were quantified by the Bradford assay, as previously described [25]. Forty $\mu \mathrm{g}$ of protein from each sample was loaded onto a sodium dodecyl sulphate-polyacrylamide gel (12\%) in a minigel apparatus (Bio-Rad) and transferred to a Polyvinylidene Fluoride (PVDF) membrane (Roche). To ensure transfer of proteins from the gel, the membrane was stained with Ponceau red. The membrane was then washed, blocked with $5 \%(\mathrm{wt} / \mathrm{vol})$ blocking agent (non-fat skimmed milk), and incubated with a primary antibody against Omp33 obtained from mouse (INIBIC, A Coruña). Proteins were visualized by incubation with horseradish peroxidase-conjugated secondary antibody, followed by enhanced chemiluminescence ECL Plus (Amersham Pharmacia Biotech) and detected with the LAS3000 chemiluminescence detector (Fujifilm).

\footnotetext{
Acknowledgements and Funding

The present study was supported by grants from SERGAS (PS08/24 and PS07/90) and INCITE 08CSA064916PR from the Xunta de Galicia, by the Spanish Network for Research in Infectious Diseases RD06/0008/0025, and by grants PI081613 and PS09/00687 from the Instituto de Salud Carlos III (Madrid).

J. Aranda is in receipt of a Sara Borrell post-doctoral grant from the Instituto de Salud Carlos III (Madrid). M. Poza and B. Gómez are in receipt of Isidro Parga Pondal postdoctoral grants from the Xunta de Galicia. S. Rumbo and C. Rumbo are in receipt of pre-doctoral grants from the Instituto de Salud Carlos III (Madrid).
}

\section{Author details}

${ }^{1}$ Servizo de Microbioloxía-INIBIC, Complexo Hospitalario Universitario A Coruña, As Xubias s/n, 15006. A Coruña, Spain. ${ }^{2}$ Departamento de Xenética. Facultade de Veterinaria. Universidade de Santiago de Compostela. Campus de Lugo. 27002 Lugo, Spain.

\section{Authors' contributions}

JA and MP conceived the design of the study, carried out several experimental procedures, and drafted the manuscript. BG and SR participated in the mutant construction and complementation. CR and JR carried out the protein analysis. PR carried out the construction of pET-RA plasmid. GB participated in the design and coordination of the study and helped to draft the manuscript. All authors read and approved the final manuscript.

Received: 9 August 2010 Accepted: 9 November 2010

Published: 9 November 2010

\section{References}

1. Munoz-Price LS, Weinstein RA: Acinetobacter infection. N Engl J Med 2008 358(12):1271-1281

2. Peleg AY, Seifert H, Paterson DL: Acinetobacter baumannii: emergence of a successful pathogen. Clin Microbiol Rev 2008, 21(3):538-582.

3. Naiemi NA, Duim B, Savelkoul PH, Spanjaard L, de Jonge E, Bart A, Vandenbroucke-Grauls CM, de Jong MD: Widespread transfer of resistance genes between bacterial species in an intensive care unit: implications for hospital epidemiology. J Clin Microbiol 2005, 43(9):4862-4864.

4. Fournier PE, Richet $\mathrm{H}$ : The epidemiology and control of Acinetobacter baumannii in health care facilities. Clin Infect Dis 2006, 42(5):692-699.

5. Coyne S, Guigon G, Courvalin P, Perichon B: Screening and quantification of the expression of antibiotic resistance genes in Acinetobacter baumannii with a microarray. Antimicrob Agents Chemother 2010, 54(1):333-340.

6. Smith MG, Gianoulis TA, Pukatzki S, Mekalanos JJ, Ornston LN, Gerstein M, Snyder M: New insights into Acinetobacter baumannii pathogenesis revealed by high-density pyrosequencing and transposon mutagenesis. Genes Dev 2007, 21(5):601-614.

7. Adams MD, Goglin K, Molyneaux N, Hujer KM, Lavender H, Jamison J, MacDonald IJ, Martin KM, Russo T, Campagnari AA, et al: Comparative genome sequence analysis of multidrug-resistant Acinetobacter baumannii. J Bacteriol 2008, 190(24):8053-8064.

8. Vallenet $D$, Nordmann P, Barbe V, Poirel L, Mangenot S, Bataille E, Dossat C, Gas S, Kreimeyer A, Lenoble $P$, et al: Comparative analysis of Acinetobacters: three genomes for three lifestyles. PLoS One 2008, 3(3): e1805.

9. Fournier PE, Vallenet D, Barbe V, Audic S, Ogata H, Poirel L, Richet $H$, Robert C, Mangenot S, Abergel C, et al: Comparative genomics of multidrug resistance in Acinetobacter baumannii. PLoS Genet 2006, 2(1):e7.

10. Heritier C, Poirel L, Lambert T, Nordmann P: Contribution of acquired carbapenem-hydrolyzing oxacillinases to carbapenem resistance in Acinetobacter baumannii. Antimicrob Agents Chemother 2005, 49(8):3198-3202

11. Choi AH, Slamti L, Avci FY, Pier GB, Maira-Litran T: The pgaABCD locus of Acinetobacter baumannii encodes the production of poly-beta-1-6-Nacetylglucosamine, which is critical for biofilm formation. J Bacteriol 2009, 191(19):5953-5963.

12. Roca I, Marti S, Espinal P, Martinez P, Gibert I, Vila J: CraA, a major facilitator superfamily efflux pump associated with chloramphenicol resistance in Acinetobacter baumannii. Antimicrob Agents Chemother 2009, 53(9):4013-4014

13. Camarena L, Bruno V, Euskirchen G, Poggio S, Snyder M: Molecular mechanisms of ethanol-induced pathogenesis revealed by RNAsequencing. PLOS Pathog 6(4):e1000834.

14. de Vries J, Wackernagel W: Integration of foreign DNA during natural transformation of Acinetobacter sp. by homology-facilitated illegitimate recombination. Proc Natl Acad Sci USA 2002, 99(4):2094-2099.

15. Soares NC, Cabral MP, Parreira JR, Gayoso C, Barba MJ, Bou G: 2-DE analysis indicates that Acinetobacter baumannii displays a robust and versatile metabolism. Proteome Sci 2009, 7:37.

16. Kato $C$, Ohmiya $R$, Mizuno $T$ : A rapid method for disrupting genes in the Escherichia coli genome. Biosci Biotechnol Biochem 1998, 62(9):1826-1829.

17. Reyrat JM, Pelicic V, Gicquel B, Rappuoli R: Counterselectable markers: untapped tools for bacterial genetics and pathogenesis. Infect Immun 1998, b66(9):4011-4017.

18. Steyert SR, Pineiro SA: Development of a novel genetic system to create markerless deletion mutants of Bdellovibrio bacteriovorus. Appl Environ Microbiol 2007, 73(15):4717-4724. 
19. Geng SZ, Jiao XA, Pan ZM, Chen XJ, Zhang XM, Chen X: An improved method to knock out the asd gene of Salmonella enterica serovar Pullorum. J Biomed Biotechnol 2009, 2009:646380.

20. Edwards RA, Keller LH, Schifferli DM: Improved allelic exchange vectors and their use to analyze 987P fimbria gene expression. Gene 1998, 207(2):149-157.

21. Ried JL, Collmer A: An nptl-sacB-sacR cartridge for constructing directed, unmarked mutations in gram-negative bacteria by marker exchangeeviction mutagenesis. Gene 1987, 57(2-3):239-246.

22. Saballs M, Pujol M, Tubau F, Pena C, Montero A, Dominguez MA, Gudiol F, Ariza J: Rifampicin/imipenem combination in the treatment of carbapenem-resistant Acinetobacter baumannii infections. J Antimicrob Chemother 2006, 58(3):697-700.

23. Fernandez-Cuenca F, Pascual A, Ribera A, Vila J, Bou G, Cisneros JM, Rodriguez-Bano J, Pachon J, Martinez-Martinez L: [Clonal diversity and antimicrobial susceptibility of Acinetobacter baumannii isolated in Spain. A nationwide multicenter study: GEIH-Ab project (2000)]. Enferm Infecc Microbiol Clin 2004, 22(5):267-271.

24. Miller JH: A short course in bacterial genetics. Cold Spring Harbor Laboratory Press, Cold Spring Harbor, NY; 1992.

25. Bradford MM: A rapid and sensitive method for the quantitation of microgram quantities of protein utilizing the principle of protein-dye binding. Anal Biochem 1976, 72:248-254.

doi:10.1186/1471-2180-10-279

Cite this article as: Aranda et al:: A rapid and simple method for constructing stable mutants of Acinetobacter baumannii. BMC Microbiology 2010 10:279.

\section{Submit your next manuscript to BioMed Central} and take full advantage of:

- Convenient online submission

- Thorough peer review

- No space constraints or color figure charges

- Immediate publication on acceptance

- Inclusion in PubMed, CAS, Scopus and Google Scholar

- Research which is freely available for redistribution

Submit your manuscript at www.biomedcentral.com/submit
Ciomed Central 